\title{
$P=i$
}

NASA Contractor Report 195005

ICASE Report No. 94-92

34449
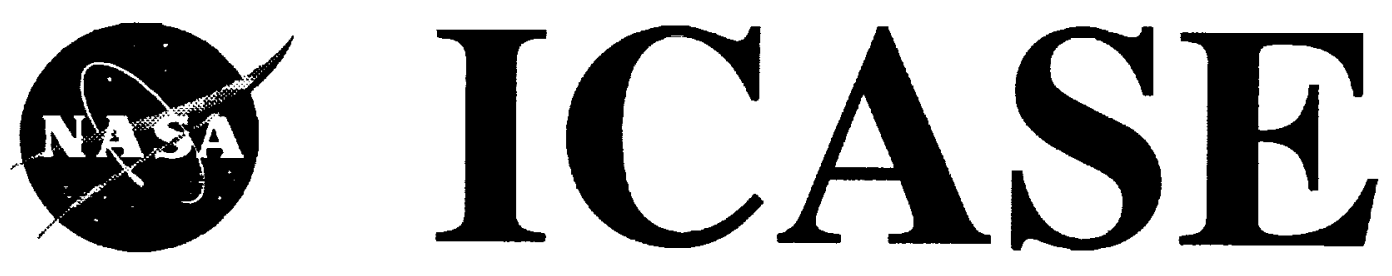

\section{ON THE LIGHTHILL RELATIONSHIP AND SOUND GENERATION FROM ISOTROPIC TURBULENCE}

\section{Ye Zhou}

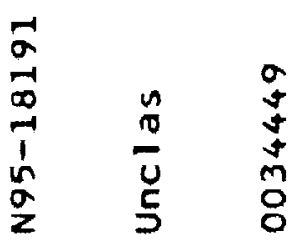

Alexander Praskovsky Steven Oncley

Contract NAS1-19480

November 1994

Institute for Computer Applications in Science and Engineering NASA Langley Research Center Hampton, VA 23681-0001

SRA Operated by Universities Space Research Association

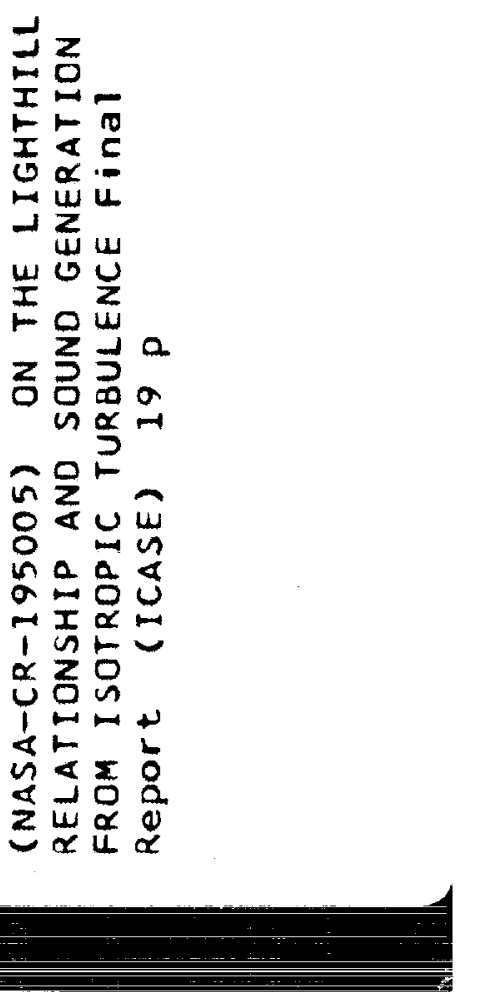




\title{
On the Lighthill relationship and sound generation from isotropic turbulence
}

\author{
Ye Zhou ${ }^{1}$ \\ Institute for Computer Applications in Science and Engineering \\ NASA Langley Research Center \\ Hampton, Virginia 23681 \\ Alexander Praskovsky and Steven Oncley \\ Atmospheric Technology Division \\ National Center for Atmospheric Research ${ }^{2}$ \\ Boulder, Colorado 80707-3000
}

\begin{abstract}
In 1952, Lighthill (Proc. Roy. Soc. A211, (1952)) developed a theory for determining the sound generated by a turbulent motion of a fluid. With some statistical assumptions, Proudman (Proc. Roy. Soc. A211, (1952)) applied this theory to estimate the acoustic power of isotropic turbulence. Recently, Lighthill established a simple relationship that relates the fourth-order retarded time and space covariance of his stress tensor to the corresponding second-order covariance and the turbulent flatness factor, without making statistical assumptions for a homogeneous turbulence. Lilley (Submitted to Theoretical and Computational Fluid Dynamics, 1993) revisited the Proudman's work and applied the Lighthill relationship to directly evaluate the radiated acoustic power from isotropic turbulence. After choosing the time separation dependence in the two-point velocity time and space covariance based on the insights gained from direct numerical simulations, Lilley concluded that the Proudman constant is determined by the turbulent flatness factor and the second-order spatial velocity covariance. In order to estimate the Proudman constant at high Reynolds numbers, we analyzed a unique data set of measurements in a large wind tunnel and atmospheric surface layer that covers a range of the Taylor microscale based Reynolds number $2.0 \times 10^{3} \leq R_{\lambda} \leq 12.7 \times 10^{3}$. Our measurements demonstrate that the Lighthill relationship is a good approximation, providing additional support to Lilley's approach. The flatness factor is found between $2.7-3.3$ and the second order spatial velocity covariance is obtained. Based on these experimental data, the Proudman constant is estimated to be $0.68-3.68$.
\end{abstract}

\footnotetext{
${ }^{1}$ This research was supported by the National Aeronautics and Space Administration under NASA Contract No. NAS1-19480 while the first author was in residence at the Institute for Computer Applications in Science and Engineering (ICASE), NASA Langley Research Center, Hampton, VA, 23681.

${ }^{2}$ The National Center for Atmospheric Research is sponsored by the National Science Foundation.
} 
I! 
Lighthill's 1952 paper "On sound generated aerodynamically" formed the theoretical foundation of modern aeroacoustics. In particular, Lighthill (1952) established an exact theory on the generation of sound by a small patch of turbulence within a very much larger but quiescent volume of fluid. To estimate the amount of sound generated by this turbulent motion, information is required concerning mean values of certain fluctuating quantities, such as the fourth-order retarded time and space covariance of the Lighthill stress tensor. It is often convenient to approximate certain types of turbulent flow as if it were isotropic since isotropic turbulence has no preferred direction and requires a minimum number of quantities to describe its structure and behavior (Hinze, 1959). The estimation of the sound radiation from isotropic turbulence is an important step in the application of Lighthill's theory and was done first by Proudman (1952). Regarding the turbulence as incompressible and at very high Reynolds number, Proudman (1952) suggested that the theory of aerodynamic noise, in the case of isotropic turbulence, is best applied to the case where the intensity of the turbulence is maintained constant in time during the processes of generation and radiation of the sound to the far-field.

Recently, Sarkar and Hussaini (1993) utilized a hybrid direct numerical simulation approach which combines direct numerical simulation (DNS) of the turbulent flow with the Lighthill acoustic analogy. They found that the acoustic power in the simulations is proportional to $u^{8} / L C_{\infty}$, where $u$ is the characteristic velocity of the energy containing eddies, $L$ is the integral scale of the turbulence, and $C_{\infty}$ is the ambient speed of the sound. This is in agreement with the analytical result of Proudman (1952), but the constant of proportionality $\alpha$ (hereafter, called the Proudman constant) is smaller than found from the analytical results quoted by Proudman for different turbulent models. The DNS computation has the advantage of being free of any assumption or modeling. Unfortunately, computing the fourth time derivative of the Lighthill stress tensor requires high orders of resolution in both space and time, and hence an absolute accuracy cannot be placed on these DNS results (Lilley, 1993). The DNS result is also restricted to moderate to small Reynolds numbers. 
Lilley (1993) revisited the original work of Proudman (1952) with particular attention paid to the Proudman's assumption that for isotropic turbulence in near incompressible flow the effects of retarded time difference can be neglected. This approximation replaces the fourth-order retarded-time and space covariance of Lighthill's stress tensor, $T_{i j}$, by the equivalent simultaneous covariance. Lilley demonstrated that although this assumption is a valid approximation in the evaluation of the two point covariance of $\partial^{2} T_{i j} / \partial t^{2}$ at low Mach number, it is not justified when Gaussian statistics are assumed in order to reduce it to the sum of products of the derivatives of equivalent second-order velocity covariances (known as the Millionshtchikov hypothesis, see Batchelor 1953). Lilley found that relaxing this assumption, however, only leads to a small change in the numerical result for the total acoustic power.

The Millionshtchikov hypothesis (Batchelor, 1953) used by Proudman (1952) four decades ago is obviously a crude assumption. Applying Lighthill's (1952) theory, Proudman (1952) found that the total sound power per unit volume in the radiated sound field is

$$
p_{s}=\frac{\rho_{\infty}}{4 \pi C_{\infty}^{5}} \int U d \mathbf{r}
$$

where $\rho_{\infty}$ is the constant density throughout the turbulent flow and in the radiation domain outside it, and the effective acoustic source is

$$
U=\frac{\partial^{4}}{\partial \tau^{4}} P_{x x, x x}(\mathbf{r})
$$

with

$$
P_{x x, x x}(\mathbf{r})=<\left(u_{A}^{2} u_{B}^{2}-<u^{2}>^{2}\right)>
$$

Here $u_{A}$ and $u_{B}$ are the components of the velocity at $\mathbf{A}$ and $\mathbf{B}$ respectively in the direction of the far-field observer at $\mathbf{x}$; and $\mathbf{r}$ and $\tau$ are the separation distance and retarded time separation. Turbulence is assumed pseudo-stationary. Lighthill (1993) elegantly showed how to relate the fourth-order retarded time and space covariance to a second order time 
and space covariance. We now briefly review the derivation in Lighthill (1993). First, one introduces the two-point correlation coefficient,

$$
R=\frac{<u(\mathbf{x}) u(\mathbf{x}+\mathbf{r})>}{<u^{2}>}
$$

assuming that both $u(\mathbf{x})$ and $u(\mathbf{x}+\mathbf{r})$ have the same mean square $\left\langle u^{2}\right\rangle$. Here $R$ represents the fraction of the variation of $u(\mathbf{x}+\mathbf{r})$ which follows that of $u(\mathbf{x})$. This suggests that we may use $u(\mathbf{x})$ as one random variable and introduce

$$
w=\frac{u(\mathbf{x}+\mathbf{r})-R u(\mathbf{x})}{\left(1-R^{2}\right)^{1 / 2}}
$$

as another, but independent, random variable with

$$
<u^{2}(\mathbf{x}+\mathbf{r})>=R^{2}<u^{2}>+\left(1-R^{2}\right)<w^{2}>
$$

so that, $\left\langle u^{2}\right\rangle=\left\langle w^{2}\right\rangle$. Based on these assumptions, we have

$$
\begin{aligned}
<u^{2}(\mathbf{x}+\mathbf{r}) u^{2}(\mathbf{x})> & =<u^{2}(\mathbf{x})\left[R u(\mathbf{x})+\left(1-R^{2}\right)^{1 / 2} w\right]^{2}> \\
& =R^{2}<u^{4}>+\left(1-R^{2}\right)<u^{2}><w^{2}> \\
& =R^{2}<u^{4}>+\left(1-R^{2}\right)<u^{2}>^{2}
\end{aligned}
$$

so that the covariance of $u^{2}(\mathbf{x})$ with $u^{2}(\mathbf{x}+\mathbf{r})$ is,

$$
\frac{<u^{2}(\mathbf{x}) u^{2}(\mathbf{x}+\mathbf{r})>-<u^{2}(\mathbf{x})>^{2}}{<u(\mathbf{x}) u(\mathbf{x}+\mathbf{r})>^{2}(\kappa-1)}=1,
$$

where the turbulent flatness factor is

$$
\kappa=\frac{<u^{4}>}{<u^{2}>^{2}}
$$

For homogeneous turbulence where $u(\mathbf{x})$ and $u(\mathbf{x}+\mathbf{r})$ have the same mean square $\left\langle u^{2}\right\rangle$, this relationship involves no statistical assumptions, and reduces to the Millionshtchikov relation when the joint probability function is Gaussian. This remarkable simple relation provides a crucial step for calculating the efficiency of the acoustic power output. 
Indeed, one now needs only to provide a correct second-order spatial-temporal covariance for a given range of Reynolds numbers. Using (4), Lilley (1993) found that

$$
P_{x x, x x}(\mathbf{r}, \tau, t)=(\kappa-1)<u^{2}>^{2}\left(f(r, \tau) \cos ^{2} \theta+g(r, \tau) \sin ^{2} \theta\right)^{2}
$$

In (6), $\theta$ is the angle between the propagation direction $\mathbf{x}$ and the vector distance $\mathbf{r}$ separating the points $\mathbf{A}$ and $\mathbf{B}$. Moreover, $f$ and $g$ are the second-order longitudinal and lateral velocity covariance. respectively.

Lilley (1993) further assumed $f(r, \tau, t)$ is in its self-preserving form, which is independent of time during the decay. In particular, the time separation dependence in $f(r, \tau, t)$ has been chosen to establish a far-field sound spectrum found in DNS. This is a plausible assumption based on the realization that the temporal covariance was very dependent on the large-eddy contribution (Lilley, private communication, 1994). For isotropic turbulence, $f(r)$ and $g(r)$ are simply related by

$$
f(r)+\frac{r}{2} \frac{\partial f(r)}{\partial r}=g(r)
$$

and an experimental verification is given by Von Kaŕmań and Howarth (1938).

Finally, Lilley (1993) found that the total acoustic power is given by

$$
p_{s}(t)=\alpha \frac{\rho_{\infty} u^{8}}{L C_{\infty}^{5}} .
$$

The Proudman constant $\alpha$ is given by

$$
\alpha=\frac{32}{15}(\kappa-1) S_{T}^{4} \Phi
$$

where the turbulent Strouhal number

$$
S_{T}=\Omega L / u
$$


is of order unity. The factor $\Phi$ is dependent on the spatial longitudinal velocity correlation covariance, $f(r / L)$, and Lilley(1993) found in general,

$$
\Phi=\int_{0}^{\infty} x^{4}\left(\frac{d f}{d x}\right)^{2} d x
$$

The turbulent Strouhal number is likely to have values in the range 1.0 to 1.25 (Lilley, 1993). Indeed, the definition of the Strouhal number, $S_{T}$, indicates that it very nearly equals unity. Since our experimental results only have $u$ and $L$, we must assume $1 / \Omega=u / L$ with $S_{T}$ equal to unity.

Thus, Lilley (1993) concluded that the Proudman constant depends critically on the shape of the longitudinal velocity correlation, $f(r)$, and the flatness factor for a given range of Reynolds numbers.

It is clear that Lilley's direct evaluation of the total acoustic power is a rational approach for establishing the base-line values for the Proudman constant. Lilley (1993) stated that in the derivation of the Lighthill relationship no statistical assumptions are involved. The Lighthill relationship has been confirmed by Sarkar (private communication, 1994) in his low Reynolds number homogeneous isotropic turbulence simulations. Lilley (1993) also presented the result of Dubois which indicates the Lighthill relation is also quite accurate in a low Reynolds number DNS with forcing at small wavenumber modes. However, the most important application of the Lighthill theory to isotropic turbulence, of course, is at the high Reynolds numbers. Thus, it is important to validate the Lighthill relationship and to estimate the Proudman constant using reliable systematic experimental data at high enough Reynolds numbers. We do so by analyzing a unique data base obtained from measurements in a large wind tunnel and atmospheric observations.

Measurements in the atmospheric surface layer were taken over a flat field near Carpenter, Wyoming, in 1990. A description of the experiment can be found in Oncley (1992). As a part of this experiment, the instantaneous horizontal component of the wind velocity was measured from a tower at $7 \mathrm{~m}$ above ground level. A standard hot-wire anemometer 
was used with a single tungsten wire $5 \mu \mathrm{m}$ in diameter and $1 \mathrm{~mm}$ length at an overheat ratio of 1.7. An analog differentiator was used to produce the velocity time derivative. The signals from the anemometer and differentiator were digitized in real-time at a sampling frequency of $9.6 \mathrm{kHz}$ and recorded on magnetic tape. The total sampling time is about 600 hours. After preliminary processing, six time series have been chosen from the total record (Praskovsky and Oncley 1994). The choice was based on two criteria: i) stationary wind speed and direction, and ii) low turbulence intensity. The quality of the chosen time series (based on the above criteria) is similar to that of wind tunnel measurements.

The second set of data was obtained in the large wind tunnel of the Central Aerohydrodynamic Institute (Moscow, Russia). Longitudinal and lateral velocity components were recorded in the mixing layer and in the return channel of the wind tunnel. The wind tunnel has an open working section $24 \mathrm{~m}$ long. The mixing layer between a jet issuing from an elliptical nozzle $\left(14 \times 24 \mathrm{~m}^{2}\right)$ and ambient air was studied. Measurements were taken at a distance $20 \mathrm{~m}$ from the nozzle edge directly downstream of the nozzle side wall. The free jet velocity was equal to $11.8 \mathrm{~m} / \mathrm{sec}$. The return channel of the wind tunnel is $175 \mathrm{~m}$ long and $22 \mathrm{~m}$ wide, and its height rises linearly from $20 \mathrm{~m}$ to $32 \mathrm{~m}$. Measurements were taken from a tower in the plane of symmetry at $5 \mathrm{~m}$ above floor level. Standard hotwire anemometers were used. An X-wire probe, with perpendicular wires, was operated at an overheat ratio of 1.8. Wires were made of platinum-plated tungsten with diameter $2.5 \mu \mathrm{m}$. Both the active length and the distance between the wires were $0.5 \mathrm{~mm}$. Data were recorded with a sampling frequency of $16 \mathrm{kHz}$. More details about this experiment and analysis of the measurement errors can be found in Karyakin et al. (1991), and Praskovsky et al. (1993).

The main flow characteristics of the measurements are listed in Table 1. The abbreviations RC, ML, and ASL denote the return channel, mixing layer, and atmospheric surface layer, respectively, and numerals after ASL correspond to the sequence of the time series. $U$ denotes the mean longitudinal velocity, $\sigma_{\phi}$ denotes the rms value of any quantity $\phi$. The Taylor microscale $\lambda$, and the Reynolds number $R_{\lambda}$, are defined with standard formulas: 
$\lambda=\sigma_{u} / \sigma_{\partial u / \partial x}, R_{\lambda}=\sigma_{u} \lambda / \nu$ where $\nu$ is the kinematic viscosity. Taylor's hypothesis was used to convert from temporal to spatial coordinates.

It is seen from figure 1 that measured values of

$$
\xi(r)=\frac{\left\langle u^{2}(\mathbf{x}) u^{2}(\mathbf{x}+\mathbf{r})>-<u^{2}(\mathbf{x})\right\rangle^{2}}{<u(\mathbf{x}) u(\mathbf{x}+\mathbf{r})>^{2}(\kappa-1)}
$$

are quite close to unity for all measurements at very high Reynolds numbers. This result demonstrates that the Lighthill relation, a remarkable simple expression that depends on the large-scale parameters, can be considered as universal at sufficiently high Reynolds number. It is well-known that the large-scale turbulence in shear flows is strongly anisotropic. While the deviations from anisotropy of turbulence are present in all other measured datasets, turbulence in the return channel (RC) is almost isotropic. With its mean shear almost zero and a huge large-small scale separation, it resembles the ideal grid turbulence (Karyakin et al. 1991). Fig. 1 shows that the Lighthill relation is indeed very accurate for the RC data.

It is evident from Table 2 that the values of the flatness factor from high Reynolds number experimental data sets are $2.7-3.3$. The measured velocity flatness factor is around 3 in the DNS of Sarkar \& Hussaini (1993) at $64^{3}$ and $128^{3}$ resolutions. Lilley (1993) used $\kappa=3$ in his model calculation, based on an earlier experiment of Townsend (1956).

The spatial longitudinal velocity correlation function $f(r)$ is shown in figure 2. Also shown in figure 2 are the model Gaussian and exponential distributions of $f(r)$. The Gaussian distribution was used by Proudman (1952); and it was also chosen by Lilley (1993) because its fair fit with low Reynolds number DNS at $r / L<1$. This figure shows that, generally speaking, the exponential distribution seems to be a better fit at high Reynolds number. Lilley (1993) also remarked that a better choice of $f(r)$ is possibly exponential distribution, based on the high Reynolds number experimental results of Stewart and Townsend (1951). 
In fact, these measured values for $f(r)$ offer an unique opportunity to computing $\Phi$ and their corresponding values for the Proudman constant numerically. We considered the contributions from the longitudinal correlation function from $r / L \leq 3$ since $f(r)$ at $r / L>3$ is dominated by the large-scale anisotropic structures, and it strongly depends on the external flow conditions. The estimates of the Proudman constant are given in Table 2 for the very high Reynolds number flows of the experiments. The measured values of $\alpha=0.68-3.68$ are in broad agreement with that of DNS by Sarkar and Hussaini (1993), $\alpha=2.6$, and that of Lilley (1993), $\alpha=3.6$, using a Gaussian distribution model for the second-order spatial longitudinal velocity correlations. Lilley (1993) noted that if $f(r)=\exp (-r / L)$ is used in place of the Gaussian distribution, the value of $\alpha$ is 3.2 .

The large eddy structure in turbulent shear flows contaminates the shape of the longitudinal correlation curve. Although the flatness factor is fairly constant, there are some variations in Proudman constant since it depends on the derivative of the longitudinal correlation function (see Eq. 11). We now show that this contamination does not lead to a large change to the estimated total acoustic power for $r / L \leq 3$. Using $f(r)$ obtained from the high Reynolds number grid generated isotropic turbulence by Stewart and Townsend for the same range of $r / L$ (Fig. 3), we repeated our calculation of the Proudman constant. Our calculation yields the values of $\Phi=0.42$ and $\alpha=2.32$, respectively. These results lie in the range of values for $\alpha$ and $\Phi$ computed from our data base obtained from measurements in a large wind tunnel and atmospheric observations (see Table 2).

From a modeling perspective, Lilley (1994, private communication) suggested that all the ASL data show a two-part structure with relatively large values of $f(r)$ at large values of $r / L$ (see detailed discussion in Townsend 1956), but falling to zero asymptotically. The longitudinal covariance could be represented by a choice of values $L 1$ and $L 2$ representing the contributions to $f(r)$ from the small and large eddies respectively. The small eddy contribution would follow an exponential law, whereas the larger eddies would follow a 'Gaussian' distribution law. 


\section{Conclusions}

We have presented measurements obtained from high Reynolds number experimental data sets in a large wind tunnel and in the atmospheric surface layer over the range of $2.0 \times 10^{3} \leq R_{\lambda} \leq 12.7 \times 10^{3}$. It is found that the Lighthill (1993) relation, which relates the fourth-order covariance to the second-order covariance and the turbulent flatness factor, is a good approximation. Therefore, Lilley's (1993) direct evaluation of the acoustic power using the Lighthill relation is supported. Lilley's work identified that the Proudman constant depends critically on the turbulent flatness factor and the second-order spatial longitudinal velocity correlation function. These parameters are determined experimentally in order to compute the Proudman constant. Our results demonstrate that the turbulent flatness is around $2.7-3.3$. It also appears that the longitudinal velocity correlation is closer to an exponential distribution than a Gaussian distribution. We evaluated the Proudman constant numerically from the experimentally measured $f(r)$ and found its values to be $0.68-3.68$.

\section{Acknowledgements}

One of the authors (Y.Z.) gratefully acknowledges the helpful discussions and comments from Prof. G.M. Lilley, Dr. J.C. Hardin, Prof. S. Sarkar and Dr. M.Y. Hussaini. 


\section{References}

Batchelor, G.K., 1953. "The theory of homogeneous turbulence", Cambridge University Press.

Hinze, J.O., 1975. "Turbulence", McGraw Hill.

Kaŕmań, T., von, and Howarth, L., 1938. "On the statistical theory of isotropic turbulence", Proc. Roy. Soc. A164, 192.

Karyakin, M. Yu., Kuznetsov, V.R., and Praskovsky, A.A., 1991. "Experimental verification of the hypothesis of fine-scale isotropy of turbulence" Fluid Dynamics, 26, 658.

Lighthill, M.J., 1952. "On sound generated aerodynamically: 1. General theory", Proc. Roy. Soc. A211, 1107.

Lighthill, M.J., 1993. "An estimate of the covariance of $T_{x x}$ without using statistical assumptions", see Appendix 1 of "On the noise radiated from a turbulent high speed jet," by Lilley, G.M. in Computational Aeroacoustics (edited by Hardin, J.C., and Hussaini, M.Y.) Springer-Verlag.

Lilley, G.M., 1993. "The radiated noise from isotropic turbulence revisited", ICASE Rep. 93-75. (Submitted to Theoretical and Computational Fluid Dynamics).

Oncley, S., 1992. "TKE dissipation measurements during the FLAT experiments," in Proc. of 10th Symp. on Turbulence and Diffusion, 165. AMS.

Praskovsky, A.A., Gledzer, E.B., Karyakin, M.Yu., and Zhou, Y., 1993. "The sweeping decorrelation hypothesis and energy-inertial scale interaction in high Reynolds number flows", J. Fluid Mech., 248, 493.

Praskovsky, A.A., and Oncley, S., 1994 "Measurements of the Kolmogorov constant and intermittency exponent at very high Reynolds numbers", Phys. Fluids 6, 2886. 
Proudman, I, 1952. "The generation of noise by isotropic turbulence," Proc. Roy. Soc. A214, 119.

Sarkar, S., and Hussaini, M.Y., 1993. "Computation of the sound generated by isotropic turbulence," ICASE Rep. 93-74. (Submitted to J. Fluid Mech.)

Stewart, R.W., and Townsend, A.A., 1951. "Similarity and self-preservation in isotropic turbulence," Phil. Trans. Roy. Soc. A243, 359.

Townsend, A.A., 1956. "The structure of turbulent shear flow," Cambridge University Press. 


$\begin{array}{lccllllll}\text { Time series } & \text { ML } & \text { RC } & \text { ASL-1 } & \text { ASL-2 } & \text { ASL-3 } & \text { ASL-4 } & \text { ASL-5 } & \text { ASL-6 } \\ U, \mathrm{~m} / \mathrm{s} & 7.87 & 10.8 & 3.25 & 6.58 & 8.10 & 7.08 & 12.9 & 14.5 \\ \sigma_{u}, \mathrm{~m} / \mathrm{s} & 1.67 & 1.03 & 0.356 & 0.693 & 1.10 & 1.00 & 1.82 & 2.08 \\ L, \mathrm{~m} & 1.3 & 4.8 & 47 & 42 & 51 & 81 & 99 & 77 \\ \lambda, \mathrm{cm} & 1.8 & 4.6 & 10.2 & 6.5 & 9.0 & 10.6 & 7.0 & 8.3 \\ R_{\lambda} \cdot 10^{-3} & 2.0 & 3.2 & 2.8 & 3.3 & 6.9 & 7.1 & 9.2 & 12.7\end{array}$

Table 1. Main flow characteristics of analysed time series.

$\begin{array}{lllllllll}\text { Time series } & \text { ML } & \text { RC } & \text { ASL-1 } & \text { ASL-2 } & \text { ASL-3 } & \text { ASL-4 } & \text { ASL-5 } & \text { ASL-6 } \\ \text { Flatness factor } & 2.8 & 3.3 & 2.8 & 2.9 & 2.8 & 2.9 & 2.7 & 2.9 \\ \Phi & 0.63 & 0.75 & 0.56 & 0.17 & 0.21 & 0.81 & 0.21 & 0.33 \\ \text { Proudman constant } & 2.40 & 3.68 & 2.14 & 0.68 & 0.79 & 3.27 & 0.75 & 1.32\end{array}$

Table 2. Measured values of the flatness factor and the Proudman constant. 


\section{Figure captions}

Figure 1. The experimental estimation of the Lighthill relationship

$$
\xi(r)=\frac{<u^{2}(\mathbf{x}) u^{2}(\mathbf{x}+\mathbf{r})>-<u^{2}(\mathbf{x})>^{2}}{<u(\mathbf{x}) u(\mathbf{x}+\mathbf{r})>^{2}(\kappa-1)}=1
$$

For symbols see Fig. 2.

Figure 2. The experimental second-order spatial longitudinal velocity correlation. Also included are the Gaussian distribution, $f(r)=e^{-\frac{\pi}{4}\left(\frac{r}{L}\right)^{2}},----;$ and the exponential distribution, $f(r)=e^{-r / L}, \longrightarrow$.

Figure 3. The experimental second-order spatial longitudinal velocity correlation from the high Reynolds number grid generated isotropic turbulence (Stewart and Townsend 1951). Solid and dashed lines are the same as in Fig. 2. 


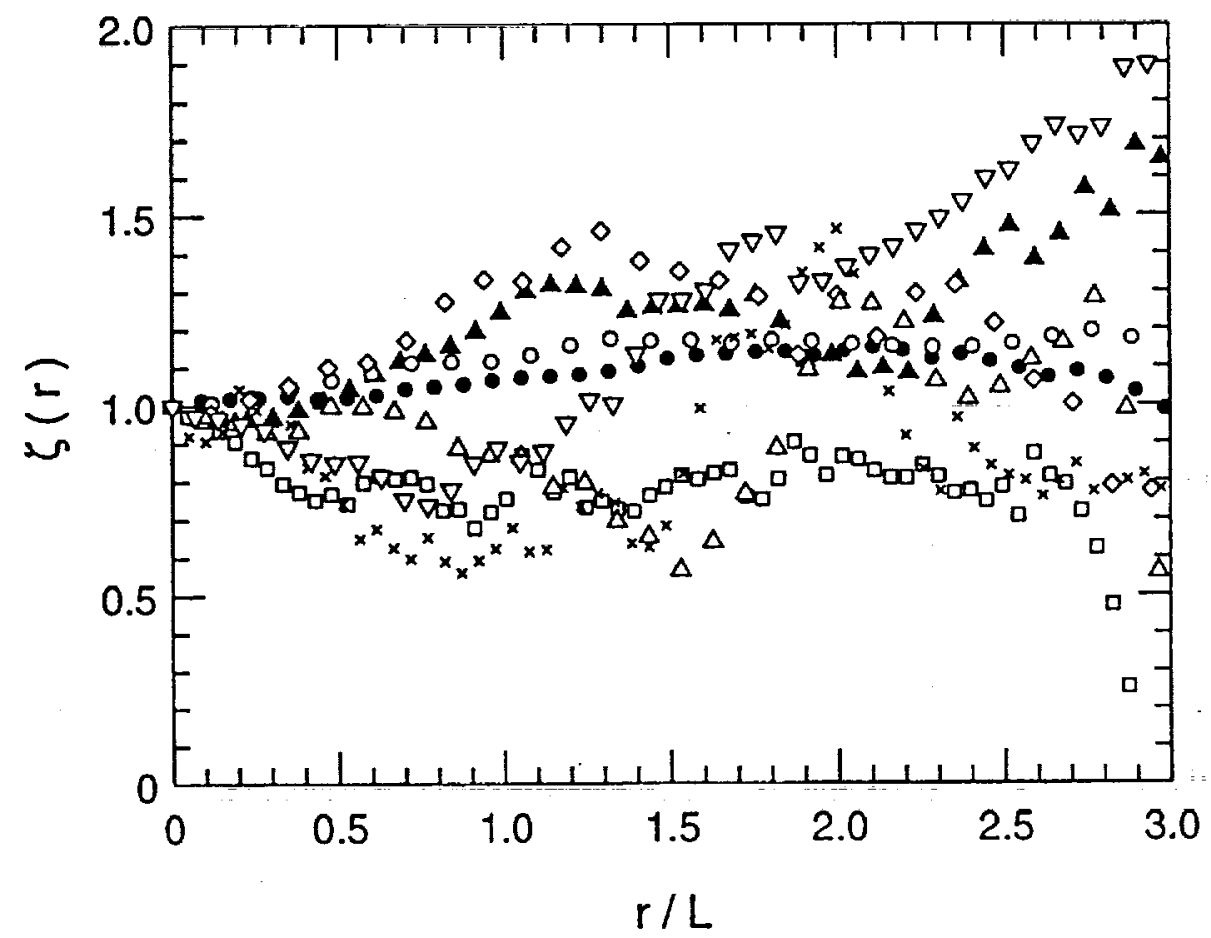

Figure 1 


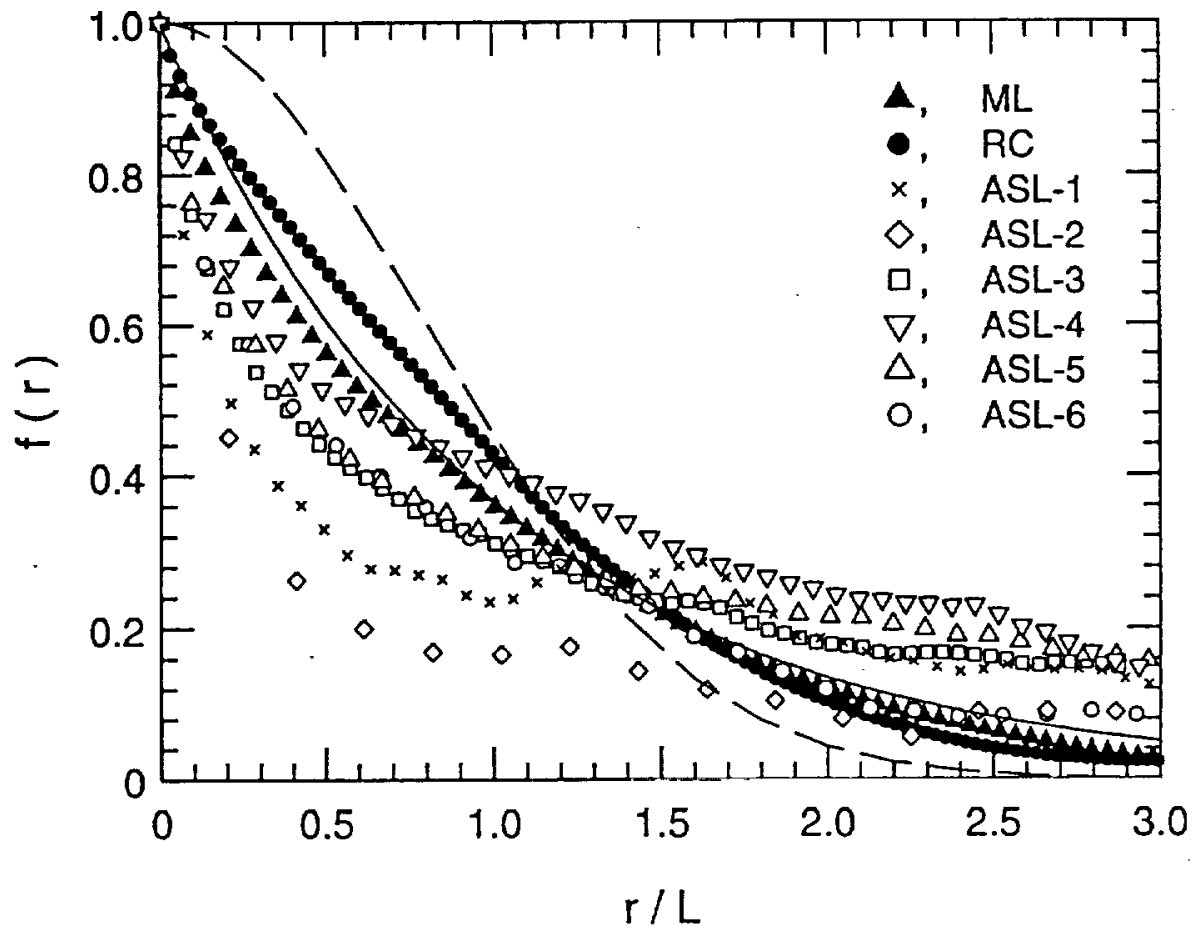

Figure 2 


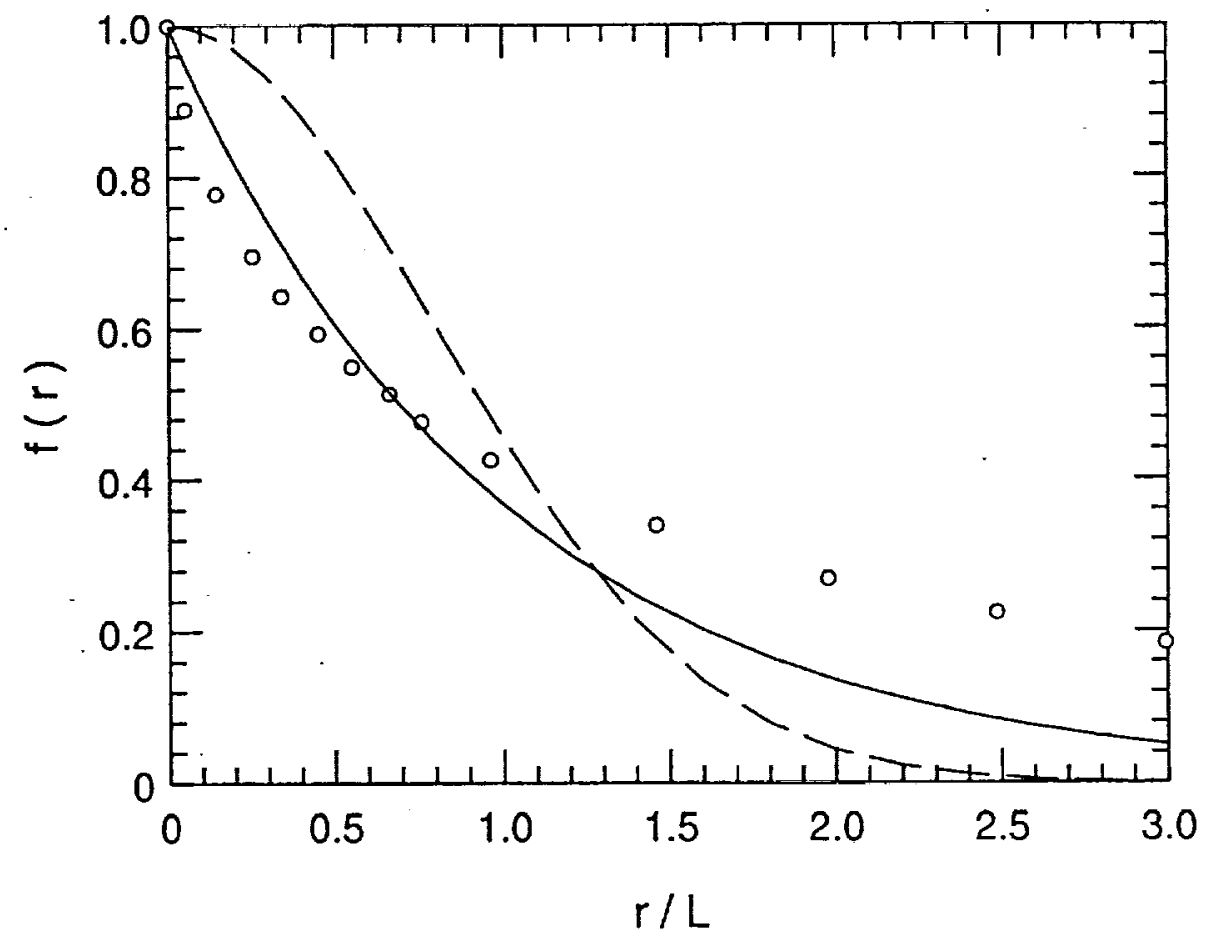

Figure 3 


\section{-}


Public reporting burden for this collection of information is estimated to average 1 hour per response, including the time for reviewing instructions, searehing existing data sources, gathering and maintaining the data needed, and completing and reviewing the collection of information. Send comments regarding this burden estimate or any other aspect of this Davis Highway, Suite 1204. Arling VA 22202-4302 and to the Office of Management and Budget, Paperwark Reduction Project (0704-0188), Washington, DC 20503.

\begin{tabular}{l|l|l} 
1. AGENCY USE ONLY(Leave blank) & $\begin{array}{l}\text { 2. REPORT DATE } \\
\text { November } 1994\end{array}$ & $\begin{array}{l}\text { 3. REPORT TYPE AND DATES COVERED } \\
\text { Contractor Report }\end{array}$ \\
\hline
\end{tabular}

4. TITLE AND SUBTITLE

ON THE LIGHTHILL RELATIONSHIP AND SOUND GENERA-

TION FROM ISOTROPIC TURBULENCE

C NAS1-19480

WU 505-90-52-01

6. AUTHOR(S)

Ye Zhou

Alexander Praskovsky

Steven Oncley

7. PERFoRMING ORgANIZATION NAME(S) AND ADDRESS(ES)

Institute for Computer Applications in Science

and Engineering

Mail Stop 132C, NASA Langley Research Center

Hampton, VA 23681-0001

9. SPONSORING/MONITORING AGENCY NAME(S) AND ADDRESS(ES)

National Aeronautics and Space Administration

Langley Research Center

Hampton, VA 23681-0001

B. PERFORMING ORGANIZATION REPORT NUMBER

ICASE Report No. 94-92

10. SPONSORING/MONITORING AGENCY REPORT NUMBER

NASA CR-195005

ICASE Report No. 94-92

\section{SUPPLEMENTARY NOTES}

Langley Technical Monitor: Michael F. Card

Final Report

To appear in Theoretical and Computational Fluid Dynamics

\begin{tabular}{l|l}
\hline 12a. DISTRIBUTION/AVAILABILITY STATEMENT & 12b. DISTRIBUTION CODE \\
Unclassified-Unlimited & \\
Subject Category 34 &
\end{tabular}

13. ABSTRACT (Maximum 200 words)

In 1952, Lighthill (Proc. Roy. Soc. A211, (1952)) developed a theory for determining the sound generated by a turbulent motion of a fluid. With some statistical assumptions, Proudman (Proc. Roy. Soc. A211, (1952)) applied this theory to estimate the acoustic power of isotropic turbulence. Recently, Lighthill established a simple relationship that relates the fourth-order retarded time and space covariance of his stress tensor to the corresponding second-order covariance and the turbulent flatness factor, without making statistical assumptions for a homogeneous turbulence. Lilley (Submitted to Theoretical and Computational Fluid Dynamics, 1993) revisited the Proudman's work and applied the Lighthill relationship to directly evaluate the radiated acoustic power from isotropic turbulence. After choosing the time separation dependence in the two-point velocity time and space covariance based on the insights gained from direct numerical simulations, Lilley concluded that the Proudman constant is determined by the turbulent flatness factor and the second-order spatial velocity covariance. In order to estimate the Proudman constant at high Reynolds numbers, we analyzed a unique data set of measurements in a large wind tunnel and atmospheric surface layer that covers a range of the Taylor microscale based Reynolds number $2.0 \times 10^{3} \leq R_{\lambda} \leq 12.7 \times 10^{3}$. Our measurements demonstrate that the Lighthill relationship is a good approximation, providing additional support to Lilley's approach. The flatness factor is found between $2.7-3.3$ and the second order spatial velocity covariance is obtained. Based on these experimental data, the Proudman constant is estimated to be $0.68-3.68$.

\begin{tabular}{|c|c|c|c|}
\hline \multicolumn{3}{|c|}{$\begin{array}{l}\text { 14. SUBJECT TERMS } \\
\text { turbulence, acoustics, noise, sound }\end{array}$} & $\begin{array}{c}\text { 15. NUMBER OF PAGES } \\
18 \\
\end{array}$ \\
\hline & & & avo \\
\hline $\begin{array}{l}\text { 17. SECURITY CLASSIFICATION } \\
\text { OF REPORT } \\
\text { Unclassified }\end{array}$ & $\begin{array}{l}\text { 18. SECURITY CLASSIFICATION } \\
\text { OF THIS PAGE } \\
\text { Unclassified }\end{array}$ & $\begin{array}{l}\text { 19. SECURITY CLASSIFICATION } \\
\text { OF ABSTRACT }\end{array}$ & $\begin{array}{l}\text { 20. LIMITATION } \\
\text { OF ABSTRACT }\end{array}$ \\
\hline
\end{tabular}

